\title{
An Overview of the Causes and Consequences of Male Fertility Decline
}

\author{
Cassie Jacobs ${ }^{1}$, Nicholas A Kerna ${ }^{1,2 *}$ and Orien L Tulp ${ }^{1}$ \\ ${ }^{1}$ College of Medicine, University of Science, Arts and Technology, Montserrat, BWI \\ ${ }^{2}$ Suriwongse Medical Center, Thailand
}

*Corresponding author: Nicholas A Kerna, College of Medicine, University of Science, Arts and Technology, 80033 USA

E-mail: nicholas.kerna@usat.edu

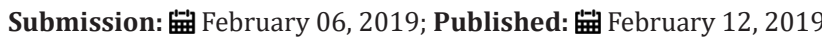

\begin{abstract}
Male infertility is a growing global public health concern. Thus, it is relevant to investigate the causes of male fertility decline and understand the consequences not only for the individual affected by male fertility decline but also the family unit and society. Causes of male fertility decline include mechanical, systems disturbances, genetic, diet, lifestyle, and environment. Consequences are far-ranging-affecting individuals, families, local societies, and population groups-with global ramifications. Formulating awareness of the causes and consequences of male fertility decline is fundamental to stimulate more specific research regarding this urgent global health issue and the prevention and treatment of male fertility decline.
\end{abstract}

Keywords: Gametogenesis; Male infertility, Microdeletion, Varicocele

Abbreviations: BMI: Body Mass Index; CAM: Complementary and Alternative Medicine; MFD: Male Fertility Decline; WHO: World Health Organization

\section{Introduction}

Globally, many men experience problems with male fertility which can affect their health, well-being, and desire to foster biological offspring. In this regard, affected males are likely to experience challenges associated with infertility, such as interpersonal relationships and to enjoy sexual intercourse to the fullest extent. The primary causes of infertility should be addressed including the frequency of male fertility decline
(MFD), primary and secondary causes of such, and associated risks to a male's overall health and quality of life. The various pharmaceutical treatments and complementary and alternative (CAM) therapies for MFD are beyond the scope of this perspective. The medical and CAM applications in MFD is an extensive study; thus, the discussion of such is left for a subsequent but associated paper.

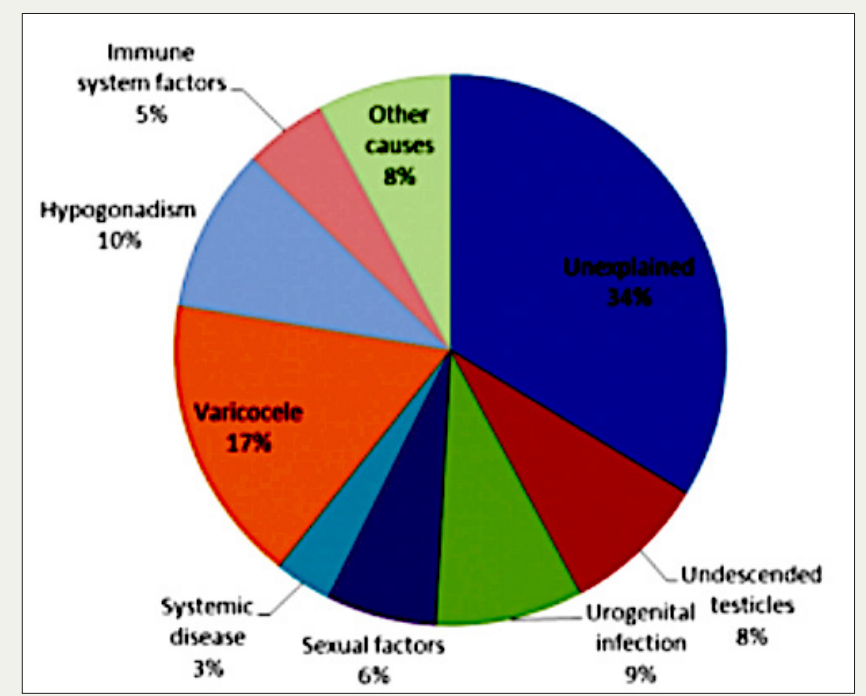

Figure 1: Causes of male fertility decline and male infertility. 


\section{Discussion}

\section{Causes of male fertility decline}

The causes of MFD (and male infertility) are numerous and varied, and may be a result of the following disorders (Figure 1):

A. varicocele

B. vesicular damage

C. hypospadias

D. cryptorchidism

E. infections within the genital tract

F. obstruction (difficulties in passing sperm from the testicles)

G. neoplasia

H. medications

I. surgery

J. testicular germ cell cancer

K. dysfunction of gametogenesis

L. immune disorders

M. endocrine disorders

N. molecular genetic factors $[1,3,4]$.

With newer investigative tools and procedures, chromosomal defects have been identified as a cause of male infertility in up to 15 percent of male infertility cases; the sex chromosomes are identified as causative in 3.8 percent and autosomes in approximately 1.3 percent of all cases [4]. Also, other abnormalities that affect RNA and molecular genetic complications include mutations in CTFR genes, KAL 1 genes, AR genes, and SHBG genes, along with a host of other genetic mutations [4]. These conditions may require genetic testing to determine if any of the abnormalities exist and to move forward with treatment to reverse the condition or to improve outcomes.

Abnormalities in the Y chromosome can cause male infertility in 15-30 percent of all cases [4]. The male $Y$ chromosome can impact male fertility as it is factorial in the development of the testes and the production of sperm during the adult years; therefore, a detailed examination of the $\mathrm{Y}$ chromosome is necessary to determine its impact on infertility in males [2]. Yd microdeletions can lead to the removal of the ASF regions of the chromosome and are common in infertile males. This key identifier is commonly evaluated during genetic screening for male infertility [2]. One out of every 12 males diagnosed as infertile has this deletion which affects approximately 10 percent of all infertile males in North America and almost 8 percent of all cases of male infertility globally [2]. Other types of deletions identified include AZFb deletions, AZFc deletions, and AZFa deletions; all of which may contribute to male infertility in some instances [2].

Proteomics can evaluate the measure of proteins in cells and make determinations regarding the changes that have occurred which may have affected fertility and its sequelae. For instance, protein expression can be measured between populations of normal versus abnormal sperm along with the identification of biomarkers to aid in a definitive diagnosis [4]. When the cause of infertility is unknown, even after semen analysis and work up, it is termed, idiopathic infertility. All of the above factors including the following lifestyle factors can contribute solely or in combination to MFD and sperm count decline which continues to fall (Figure 2).

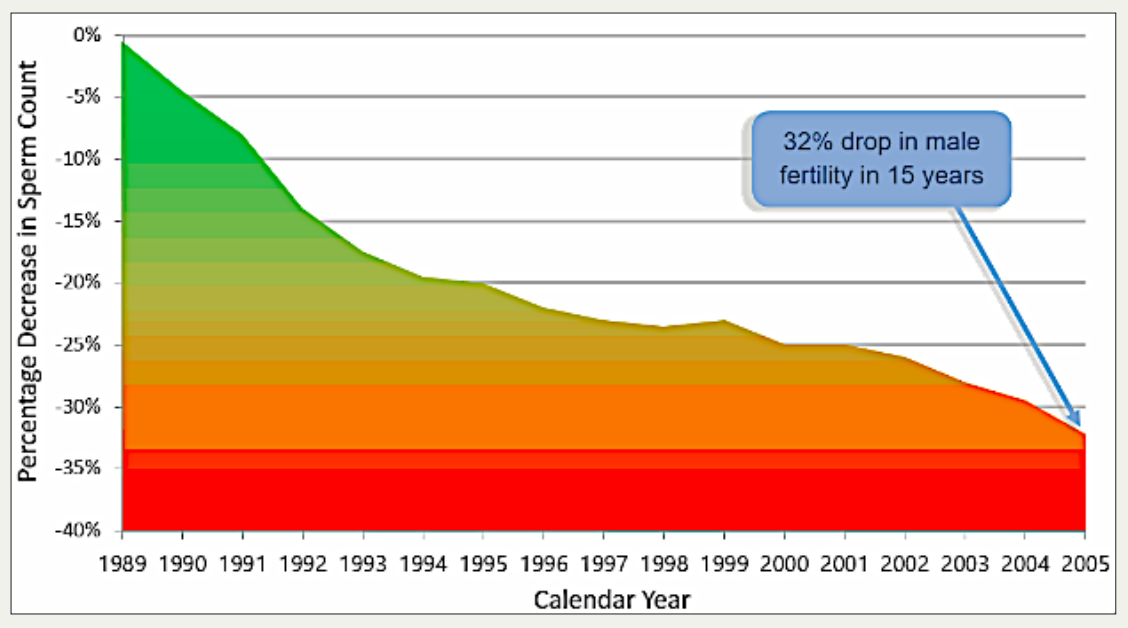

Figure 2: The percentage decrease in 35-year-old male sperm counts from 1985 to 2005.

\section{Lifestyle factors in male fertility decline}

One of the most critical factors associated with male infertility is sperm quality and quantity [5]. There are a number of factors that may impact sperm quality and quantity including the following:
A. age
B. body mass index (BMI)
C. consumption of caffeine-containing drinks and products
D. smoking (tobacco) 
E. alcohol consumption

F. environment (temperature, pollution, and toxins)

G. tight underclothing

H. prolonged sexual abstinence

I. frequency of ejaculation [5].

Lifestyle factors are major contributors to the risk of poor sperm quality and quantity in some males and can have a significant impact on fertility rates. However, it is unlikely that a single factor is to blame; instead, it may be a combination of factors that impacts this condition resulting in exacerbations toward the infertile state [5]. For many males, understanding the causes of male infertility is crucial. When the condition can be attributed to lifestyle factors, these factors can be addressed to improve outcomes and possibly reverse or, at a minimum, improve fertility in male patients [5].

\section{Consequences of male fertility decline}

According to the World Health Organization (WHO), the inability to conceive children leads to social, psychological, and severe medical consequences. MFD and male infertility can stress personal relationships and marriages and disrupt the family unit. The effects of low fertility rates are evidenced in countries such as Japan and throughout the European Union. The reduced reproduction rate is causing harm to many people and societies worldwide [3]. In developing countries, there are severe social, psychological, and economic consequences for infertile men (as well as women).

\section{Conclusion}

In the past, MFD was considered a private matter. However, now that the related consequences to MFD are being revealed, it is considered a global health issue. MFD is an under-observed but relevant public health issue. The causes of this condition are numerous and varied. Genetic factors in MFD are now coming to the forefront of research and debate.
Lifestyle factors have been shown to impact male fertility. For this reason, lifestyle factors should be further investigated; lifestyle changes can make a dramatic impact on certain causes of MFD. Dialogue regarding preventive strategies and treatments, including complementary and alternative therapies, should be encouraged. Particular societies' distinct religious and spiritual beliefs and traditions make public health policy regarding MFD a challenging proposal. Programs must be enacted in order to reverse social stigma, detrimental psychological effects, and loss of economic security that results from this condition.

\section{Conflict of Interest}

The authors declare that this paper is written in the absence of any commercial or financial relationship that could be construed as a potential conflict of interest.

\section{References}

1. Barratt CLR, Bjorndahl L, De Jonge CJ, Lamb DJ, Osorio Martini F, et al. (2017) The diagnosis of male infertility: an analysis of the evidence to support the development of global WHO guidance-challenges and future research opportunities. Hum Reprod Update 23(6): 660-680.

2. Colaco S, Modi D (2018) Genetics of the human Y chromosome and its association with male infertility Reprod Biol Endocrinol 16(1):14.

3. Skakkebaek NE, Meyts ERD, Buck Louis GM, Toppari J, et al. (2016) Male reproductive disorders and fertility trends: influences of environment and genetic susceptibility. Physiol Rev 96(1): 55-97.

4. Tahmasbpour E, Balasubramanian D, Agarwal A (2014) A multi-faceted approach to understanding male infertility: gene mutations, molecular defects and assisted reproductive techniques (ART). J Assist Reprod Genet 31(9): 1115-1137.

5. Wogatzsky J, Wirleitner B, Stecher A, Vanderzwalmen P, et al. (2012) The combination matters - distinct impact of lifestyle factors on sperm quality: a study on semen analysis of 1683 patients according to MSOME criteria. Reprod Biol Endocrinol 10: 115.
Creative Commons Attribution 4.0 International License

For possible submissions Click Here

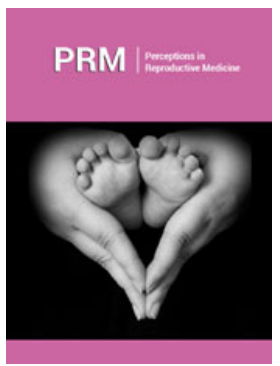

Perceptions in Reproductive Medicine

\section{Benefits of Publishing with us}

- High-level peer review and editorial services

- Freely accessible online immediately upon publication

- Authors retain the copyright to their work

- Licensing it under a Creative Commons license

- Visibility through different online platforms 